\title{
2015 AMS-SIAM Birkhoff Prize
}

EMMANUEL CANDÈs was awarded the 2015 AMSSIAM George David Birkhoff Prize in Applied Mathematics at the Joint Mathematics Meetings in San Antonio, Texas, in January 2015.

\section{Citation}

The 2015 George David Birkhoff Prize in Applied Mathematics is awarded to Emmanuel Candès for his work on compressed sensing, which has revolutionized signal processing and medical imaging, and for his related work on computational harmonic analysis, statistics, and scientific computing. In his $\mathrm{PhD}$ thesis, Candès developed curvelets and ridgelets as generalizations of wavelets that capture higher-order structure. Starting from his computational experiments with MRI images, Candès, along with coworkers Terence Tao and Justin Romberg, developed compressed sensing and introduced the Restricted Isometry Property (RIP) that leads to exact recovery of sparse signals with high probability. As some of the original work on compressed sensing, these results have had a broad impact on mathematics, statistics, and applications. Candès has continued to successfully apply principles of sparsity and $l_{1}$ minimization to a variety of other problems, such as matrix completion, robust principal component analysis, and phase retrieval (i.e., recovery of a signal from measurement only of its magnitude).

\section{Biographical Sketch}

Emmanuel Candès is the Barnum-Simons Chair in Mathematics and Statistics, a professor of electrical engineering (by courtesy), and a member of the Institute of Computational and Mathematical Engineering at Stanford University. Prior to his appointment as a Simons Chair, Candès was the Ronald and Maxine Linde Professor of Applied and Computational Mathematics at the California Institute of Technology. His research interests are in computational harmonic analysis, statistics, information theory, signal processing, and mathematical optimization with applications to the imaging sciences, scientific computing, and inverse problems. He received his $\mathrm{PhD}$ in statistics from Stanford University in 1998. Candès has received several awards, including the Alan T. Waterman Award from the National Science Foundation (NSF),

DOI: http://dx.doi.org/10.1090/noti1233 which recognizes the achievements of early-career scientists. Other honors include the 2005 James H. Wilkinson Prize in Numerical Analysis and Scientific Computing awarded by the Society for Industrial and Applied Mathematics (SIAM), the 2008 Information Theory Society Paper Award, the 2010

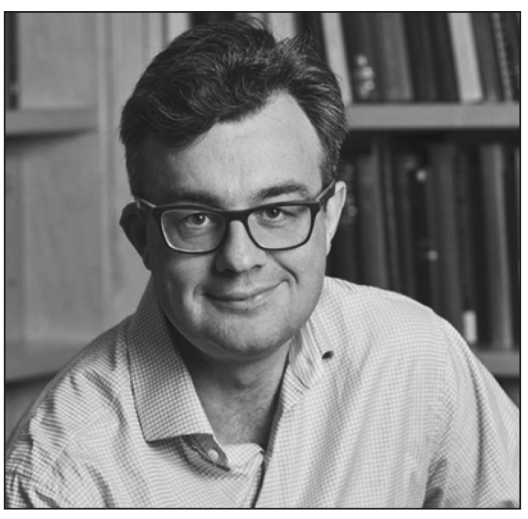

Emmanuel Candès
George Pólya Prize awarded by SIAM, the 2011 Collatz Prize awarded by the International Council for Industrial and Applied Mathematics (ICIAM), the Lagrange Prize in Continuous Optimization awarded jointly by the Mathematical Optimization Society (MOS) and SIAM, and the 2013 Dannie Heineman Prize presented by the Academy of Sciences at Göttingen. In 2014 Candès was elected to the National Academy of Sciences and to the American Academy of Arts and Sciences.

\section{Response from Emmanuel Candès}

I owe a great debt of gratitude to the American Mathematical Society and the Society for Industrial and Applied Mathematics for selecting me as the recipient of the George David Birkhoff Prize. One of the most appealing aspects of receiving a prize is that one is automatically joining the company of previous recipients. Here, I cannot think of a higher honor than being associated with those distinguished mathematicians who have all shaped our science over the last fifty years. Incidentally, I have taught the 1931 Birkhoff ergodic theorem a countless number of times. Historically, applied mathematics was largely motivated by great questions in physics. However, the applications of mathematics keep on expanding, and in the last few decades we have witnessed the explosion of a new kind of applied mathematics whose primary concern is information and data. I wish to thank Yves Meyer and David Donoho for introducing to me to the mathematics of information. David served as my $\mathrm{PhD}$ advisor in statistics at Stanford and gave me the best start any young scholar can possibly receive in any field-period. If I have any 
AMERICAN MATHEMATICAL SOCIETY MathSciNet
Mathematical Reviews

The premier gateway to scholarly literature in the mathematical sciences

Since 1940, Mathematical

Reviews (MR) has served

researchers and scholars in

the mathematical sciences by

providing timely information

on peer-reviewed articles

and books. The database of

more than 3 million items

contains reviews, abstracts,

and bibliographic information

for much of the world's

mathematical sciences

literature.



taste for problems, I owe it to him. The citation highlights joint work with Terence Tao and with my former postdoc Justin Romberg; this distinction is as much theirs at it is mine. In fact, I regard my collaboration with Terry over the years as one of the most precious things that has happened to me. Brad Efron famously said that the advantage of being a statistician is that you can play in everyone's backyard. I wish to thank the scientists and engineers (circuit designers, radiologists, biophysicists,...) who have allowed me to apply our theories, making their promise a little more precise. Finally, I have been blessed with wonderful graduate students and postdocs who have had a great diversity of backgrounds. These young collaborators, with whom I have done most of my work, have enriched me tremendously and taken me in unexpected directions. I am particularly grateful to Laurent Demanet, Lexing Ying, Justin Romberg, Benjamin Recht, Stephen Becker, Yaniv Plan, Xiaodong Li, Vladislav Voroninski, Mahdi Soltanolkotabi, Carlos Fernandez Granda, and Rina Foygel Barber for expanding my research horizons. My last words are for the love of my life, Chiara Sabatti, who has played the most important role in my professional and personal development. I married up.

\section{About the Prize}

The Birkhoff Prize recognizes outstanding contributions to applied mathematics in the highest and broadest sense and is awarded every three years. Established in 1967, the prize was endowed by the family of George David Birkhoff (1884-1944), who served as AMS President during 1925-1926. The prize is given jointly by the AMS and the Society for Industrial and Applied Mathematics (SIAM). The recipient must be a member of one of these societies. The prize carries a cash award of US\$5,000.

The recipient of the Birkhoff Prize is chosen by a joint AMS-SIAM selection committee. For the 2015 prize, the members of the selection committee were Russel Caflisch, Bjorn E. Engquist, and Avi Wigderson.

Previous recipients of the Birkhoff Prize are Jürgen K. Moser (1968), Fritz John (1973), James B. Serrin (1973), Garrett Birkhoff (1978), Mark Kac (1978), Clifford A. Truesdell (1978), Paul R. Garabedian (1983), Elliott H. Lieb (1988), Ivo Babuška (1994), S. R. S. Varadhan (1994), Paul H. Rabinowitz (1998), John N. Mather (2003), Charles S. Peskin (2003), Cathleen S. Morawetz (2006), Joel Smoller (2009), and Bjorn Engquist (2012). 